\title{
Xanthine oxidase injurious response in acute joint injury
}

\author{
Thomas Stabler $^{\text {a,* }}{ }^{\text {, Robert D. Zura }}{ }^{\mathrm{b}}$, Ming-Feng Hsueh ${ }^{\mathrm{a}}$, Virginia B. Kraus ${ }^{\mathrm{a}, \mathrm{c}}$ \\ a Duke Molecular Physiology Institute, Duke University School of Medicine, Durham, NC, USA \\ b Department of Orthopaedic Medicine, Duke University School of Medicine, Durham, NC, USA \\ c Department of Medicine, Division of Rheumatology, Duke University School of Medicine, Durham, NC, USA
}

\section{A R T I C L E I N F O}

\section{Article history:}

Received 16 February 2015

Received in revised form 28 August 2015

Accepted 24 September 2015

Available online 28 September 2015

\section{Keywords:}

Xanthine oxidase

Oxidation

Osteoarthritis

Knee injury

\begin{abstract}
A B S T R A C T
Background: While acute trauma is a major cause of osteoarthritis, its etiology is poorly understood. We sought to determine whether xanthine oxidase (XO), a major producer of reactive oxygen species, plays a role in the early events of acute joint injury.

Methods: We analyzed synovial fluid from 23 subjects with recent severe acute knee injury. As a control we evaluated SF from 23 individuals with no or minimal knee osteoarthritis. We measured XO activity, reactive oxygen + reactive nitrogen species (ROS + RNS), protein oxidative damage (carbonyl), the type II collagen synthesis marker procollagen II c-propeptide (CPII) and the type II collagen degradation marker collagen type II telopeptide (CTx-II). We also measured the proinflammatory cytokine IL-6.

Results: $\mathrm{XO}$ and ROS + RNS were higher ( $\mathrm{p}=0.02$ and $\mathrm{p}=0.001$ respectively) in acute injury than control and were strongly positively associated $(r=0.62, p=0.004)$. Carbonyl was higher in acute injury than control $(\mathrm{p}=0.0002)$ and was positively correlated with $\mathrm{XO}(\mathrm{r}=0.68, \mathrm{p}=0.0007)$ as well as with ROS $+\mathrm{RNS}(\mathrm{r}=0.71$, $\mathrm{p}=0.004)$. CPII was higher in acute injury than control $(\mathrm{p}<0.0001)$ and was negatively correlated with XO $(\mathrm{r}=-0.49, \mathrm{p}=0.017)$. While CTxII was not significantly higher in acute injury than control, it was positively correlated with CPII $(r=0.71, p=0.0002)$. IL-6 was higher in acute injury than control $(p<0.0001)$.

Conclusions: These results are consistent with a potentially injurious effect of XO activity in acute joint injury characterized by excess free radical production and oxidative damage. These effects are associated with an inhibition of type II collagen production that may impede the ability of the injured joint to repair.
\end{abstract}

(c) 2015 Elsevier B.V. All rights reserved.

\section{Introduction}

Xanthine oxidoreductase (XOR) is a widely distributed molybdoflavoenzyme that catalyzes the oxidation of hypoxanthine to xanthine and further catalyzes the oxidation of xanthine to uric acid (UA) [1]. XOR appears in 2 distinct interconvertible forms [2]: a constitutively expressed dehydrogenase form; and an oxidase form (XO) that is post-translationally modified by reversible thiol oxidation or irreversible proteolytic cleavage [3]. Circulating XOR exists almost exclusively as the proteolytic generated XO [4]. The XO form oxidizes purines to urate; thereby, it becomes a major source of reactive oxygen species (ROS) production $[5,6]$. XOR is central to the innate immune system; XO generated ROS, such as superoxide and $\mathrm{H}_{2} \mathrm{O}_{2}$, function as important second messengers in the Toll-like receptor-NF- $\kappa B$ pathway and at high concentrations play an important role in the phagocytic killing of pathogens [7]. However, high concentrations of ROS are well known to cause damage to many cell types, including synoviocytes, chondrocytes, and the extracellular matrix $[8,9]$.

\footnotetext{
* Corresponding author at: Duke University, 300 N Duke St, Durham, NC 27701, United States.

E-mail address: tvs@duke.edu (T. Stabler).
}

Although the liver and intestine are the predominant sites of XOR production in humans [10], it is expressed in significant quantities in human synovium [11]. Therefore production of XOR by synovial cells and/or other cells in joint tissues might be the source of ROS, known to be generated by tissue trauma $[5,7,12]$. This oxidative burst is thought to cause additional damage to the tissue and contribute to cell senescence [13]. Although the mechanism is not well understood, acute trauma to the knee joint is known to be a major risk factor for the future development of osteoarthritis (OA) [14]. We hypothesized that XOR, produced through activation of the innate immune response by acute trauma, plays a role in facilitating the future development of post-traumatic arthritis by serving as the source of ROS production in acute trauma. To our knowledge, this is the first study of XOR in acute joint injury in humans.

\section{Materials and methods}

\subsection{Summary: baseline characteristics of study cohort}

\begin{tabular}{llll}
\hline & $\begin{array}{l}\text { Age in years } \\
\text { mean } \pm \text { SD (range) }\end{array}$ & $\begin{array}{l}\text { Gender ratio } \\
\text { (male/female) }\end{array}$ & $\begin{array}{l}\text { Days since injury } \\
\text { mean } \pm \text { SD (range) }\end{array}$ \\
\hline Injury cohort $(\mathrm{n}=23)$ & $39.4 \pm 19.3(20-84)$ & $13 / 10$ & $11.7 \pm 7.2(1-26)$ \\
Control cohort $(\mathrm{n}=23)$ & $64.0 \pm 12.9(38-81)$ & $14 / 9$ & NA \\
\hline
\end{tabular}




\subsection{Detailed characteristics of acute injury cohort}

Samples for this study were derived from two different sources, the first being a randomized double-blinded placebo-controlled pilot trial of a single injection of short-acting intra-articular IL-1Ra (anakinra), administered to patients presenting to the Duke Sports Medicine clinic with a history of recent (within the previous month) severe knee injury due to sports injury [15]. The trial included 11 patients, 5 saline injected placebo and 6 anakinra injected study participants. We collected serum from 11 patients, 9 of which supplied matching SF samples ( 4 placebos and 5 drugs). The cohort was young and otherwise healthy with a mean age at enrollment of $23 \pm 3.5$ years and a $6 / 5$ male/female split. To exclude patients with pre-existing $\mathrm{OA}$, the study was limited to individuals $<40$ years with no prior history of joint symptoms or trauma. Patients were enrolled soon after initial injury as possible with a mean baseline enrollment time of $15.2 \pm 7.2$ days after injury (range 6-19). While synovial fluid (SF) was collected at both of these time points, only the pretreatment baseline samples were used in this study. The joint pathology was defined by clinical knee magnetic resonance images obtained prior to baseline assessments, and included, in addition to evidence of anterior cruciate ligament tear in all patients, other knee joint tissue damage including bone contusions, medial collateral ligament tears, meniscal tears and chondral defects.

The remaining SF samples were collected from patients undergoing surgery for knee joint trauma at Duke University Medical Center, and were collected at the time of surgery. This cohort had a mean age at collection of $49.5 \pm 18.6$ years and a $9 / 5$ male/female split. To exclude patients with pre-existing $\mathrm{OA}$, the opinion of the surgeon as to whether there was pre-existing OA was used. Mean collection time was $10.7 \pm 8.8$ days after injury (range $1-26$ ) with an undetermined time of injury for 4 of the subjects.

Mean age for the combined acute injury group was $39.4 \pm 19.3$ years with a $13 / 10$ male/female split and mean days after injury $11.7 \pm 7.2$ (range 1-26) with an undetermined time of injury for 4 of the subjects.

\subsection{Detailed characteristics of knee OA reference cohort}

A total of 159 participants (118 female, 41 male) were enrolled in the National Institutes of Health sponsored Prediction of Osteoarthritis (POP) study [16]. Exclusion criteria consisted of the following: exposure to a corticosteroid (either parenteral or oral) within 3 months before the study evaluation; known history of avascular necrosis, inflammatory arthritis, Paget's disease, joint infection, periarticular fracture, neuropathic arthropathy, pseudogout, or reactive arthritis. None of the participants had a history of gout in the knee, and none were taking traditional gout medications (allopurinol or colchicine) or anticoagulants. In this cohort, knee radiographic OA Kellgren-Lawrence (KL) grades [17] ranged from 1-3 for the signal knees and 0-4 for the contralateral knees. Arthrocentesis was attempted on all non-replaced knees for all participants. Sufficient SF ( $100 \mu \mathrm{l})$ was available for XO analysis of 23 specimens from knees with minimal or no OA (KL grades $0-1$ ). Theoretically, concentrations of XO activity for this reference control group would be least affected by disease and would approximate normal. This subgroup from the POP study was of similar mean age and age range (38-81 years, mean 64 years) to the POP sample as a whole (37-85 years, mean 64 years).

\subsection{Sample collection and processing}

All samples were collected with informed consent and this research was performed with the approval of the Duke University Institutional Review Board and in compliance with the Helsinki Declaration. SF was centrifuged $\left(8^{\circ} \mathrm{C}, 3500 \times \mathrm{g}, 5 \mathrm{~min}\right)$, and the supernatant aliquoted and frozen at $-80^{\circ} \mathrm{C}$ within $2 \mathrm{~h}$ of collection.

\subsection{SF analyses}

XO activity was measured using a commercially available multistep enzymatic kit per the manufacturer's directions with resorufin fluorescence as the endpoint (Cayman Chemical). Total free radical presence was measured per manufacturer's directions using the commercially available OxiSelect In Vitro ROS/RNS assay kit (Cell Biolabs) which employs a proprietary quenched fluoregenic probe that is specific for ROS plus reactive nitrogen species (RNS). As a measure of oxidative damage, protein carbonyl was quantified spectrophotometrically using a 2,4dinitrophynlhydrazine (DNPH) reaction based kit with some modifications made to the manufacturer's protocol in order to improve sensitivity and reproducibility (Cayman). Those modifications included the following: increasing the amount of sample protein to $20-40 \mathrm{mg}$; increasing the amount of DNPH to $1 \mathrm{ml}$; increasing the reaction time to $2 \mathrm{~h}$; increasing the number of pellet washes to 4; using a Beadbeater (BioSpec Products) to resuspend the protein pellet; and using a $1 \mathrm{~cm}$ pathlength spectrophotometer instead of a plate reader to measure absorbance. As indicators of type II collagen synthesis and degradation, procollagen II C-propeptide (CPII) and collagen type II telopeptide (CTx-II) were measured by competitive ELISA using commercially available kits per the manufacturer's instructions (Ibex Pharmaceuticals, and Immunodiagnostic Systems respectively). The minimum detectable concentration for the CPII kit is reported as $35.1 \mathrm{ng} / \mathrm{ml}$ with intra- and inter-assay CVs as 3.68\% and 9.08\%, respectively. The minimum detectable concentration for the CTx-II kit is reported as $0.2 \mathrm{ng} / \mathrm{ml}$ with intraand inter-assay CVs as 5.857\% and 9.967\%, respectively. The proinflammatory cytokine IL-6 was quantified using the commercially available V-plex Human IL-6 sandwich immunoassay per manufacturer's directions (Meso Scale Diagnostics). The minimum detectable concentration for IL-6 is $0.06 \mathrm{pg} / \mathrm{ml}$ with intra- and inter-assay CVs of $<5 \%$ and $<7 \%$, respectively.

\subsection{Statistical Analysis}

The Mann-Whitney nonparametric test was used to compare the concentrations of the targeted analytes in the injury cohort with the reference control group. Linear regression was used to examine the relationship of variables within a cohort. P values were adjusted by generalized linear modeling (GLM) to control for age. Analyses were performed using Graphpad Prism software and JMP statistical software from SAS.

\section{Results}

SF XO activity in the acute injury group was significantly higher $(\mathrm{p}=0.02)$ than the SF XO activity in the reference control group (Fig. 1). The SF total free radical level (ROS + RNS) was significantly higher $(p=0.001)$ in the acute injury group than the reference control group (Fig. 2A). Importantly this measure of oxidative radicals was strongly positively associated $(r=0.62, p=0.004)$ with SF XO activity (Fig. $2 \mathrm{~B}$ ) in the injury group but not in the control group $(\mathrm{r}=0.08$, $\mathrm{p}=0.71)$.

Oxidative damage to proteins, as indicated by SF carbonyl concentration (per mg protein), was significantly higher $(\mathrm{p}=0.0002)$ in the acute injury group than the reference control group (Fig. 3A). Importantly, this indirect measure of ROS production was strongly positively associated $(\mathrm{r}=0.71, \mathrm{p}=0.0004)$ with SF ROS + RNS levels (Fig. 3B) as well as $\mathrm{XO}$ activity $(\mathrm{r}=0.68, \mathrm{p}=0.007)$ in the injury group (Fig. $3 \mathrm{C}$ ) but not in the control group (for ROS + RNS: $r=-0.28, p=0.20$; for XO activity $r=0.03, p=0.89$ ).

We evaluated biomarkers indicative of collagen type II synthesis (CPII) and degradation (CTX-II). Although there was a significant positive association between SF CPII and SF CTx-II $(r=0.71, p=0.0001$, Fig. 4A), only SF CPII ( $p<0.0001$, Fig. 4B) and not CTXII was significantly higher in the acute injury group than the reference control group. In addition, only SF CPII ( $r=-0.49, \mathrm{p}=0.017$, Fig. 4 C), but not SF CTXII 


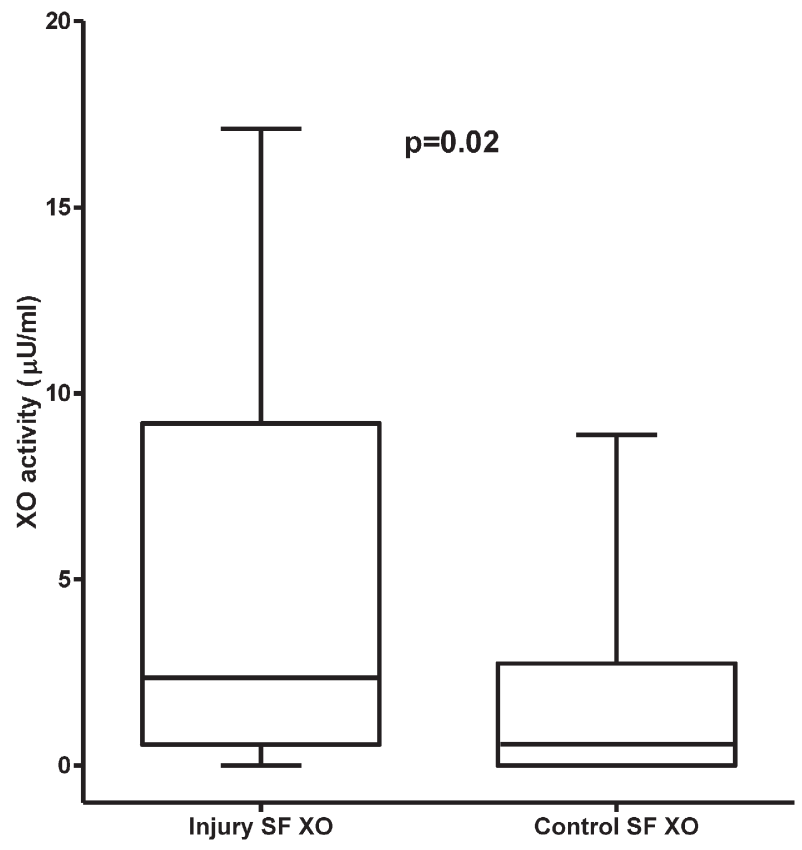

Fig. 1. Xanthine oxidase (XO) activity in synovial fluid (SF): SF XO activity was significantly higher $(\mathrm{p}=0.02)$ in the acute injury group (median $2.364 \mu \mathrm{U} / \mathrm{ml}$ ) than that in the reference control group (median $0.58 \mu \mathrm{U} / \mathrm{ml}$ ). Box plots depict median with 25 th-75th\% boxes and min to max whiskers.

$(\mathrm{r}=-0.34, \mathrm{p}=0.12)$ was negatively correlated with SF XO activity. Neither SF ROS + RNS or carbonyl content was associated with either SF CPII or SF CTx-II.

As a marker of acute inflammation, we measured the proinflammatory cytokine IL- 6 and found it to be much higher $(\mathrm{p}<0.0001)$ in the acute injury group than the reference control group (Fig. 5). No association was found between IL-6 and any of the other analytes.

\section{Discussion}

High levels of circulating XO are mostly seen with conditions involving liver damage, particularly acute viral hepatitis [18-20]; there has been at least one report of high levels of circulating XO in inflammatory rheumatic diseases [21]. In general, XO activity in normal human serum is very low [18,22]; in contrast, synovial fluid XO concentrations in acutely injured knees were very high. High XO activity in injured joints could be due to high local production and/or slow clearance. XO enzyme activity could be produced locally in the joint by the synovial cells [11] and/or the phagocytic cells infiltrating the synovium [23,24]. The second mechanism, the slower clearance of XO, is deduced from evidence that $\mathrm{XO}$ is readily immobilized and stabilized by binding to glycosaminoglycans (GAGs) $[25,26]$. Since SF, and injury SF in particular, contains very large amounts of GAGs, it is likely that XO clearance into the serum from the synovial fluid is reduced through stabilization by interaction with GAG.

Protein carbonylation is the most common form of oxidative protein modification [27]. While protein carbonylation reflects damage from many forms of ROS [28], its correlation here with SF ROS + RNS and SF XO activity, concurrent with the association of SF ROS + RNS and SF XO activity, is a strong indication that XO activity is a major contributor to ROS in the acute joint injury setting.

While ROS actually play an important role in maintaining normal cartilage function [29], an imbalance in ROS/antioxidants can lead to oxidative stress with subsequent cell senescence, cell death and possible cartilage degradation [30]. CPII is known to be directly correlated with type II collagen synthesis [31], and has been shown to increase after knee injury [32]; so the increase we observed here with injury is not surprising. The negative correlation we found between SF XO activity and SF CPII suggests that the activity of XO impairs collagen synthesis and thereby extracellular matrix repair in the context of joint injury. The repair response, reflected in the level of SF CPII, would likely have been much greater without the increase in XO activity. While we did not observe a correlation of SF ROS + RNS or SF carbonyl content with SF CPII, it is quite possible that the reduction in cartilage synthesis in association with increasing XO activity is acting instead through some other system. It has been shown that XO induces apoptosis in human chondrocytes, which are the source of CPII [33]. While this induction is thought to be due to ROS production from XO, it is possible that it is operating through other means, which would explain the lack of association found between CPII and ROS + RNS or carbonyl. While other studies have shown an increase in cartilage degradation with joint trauma as measured by CTx-II [31,34,35], we found no such increase. It is probable that the acute timepoint of most of our injury samples (mean 12 days) is the most likely explanation as collagen degradation products begin to
A

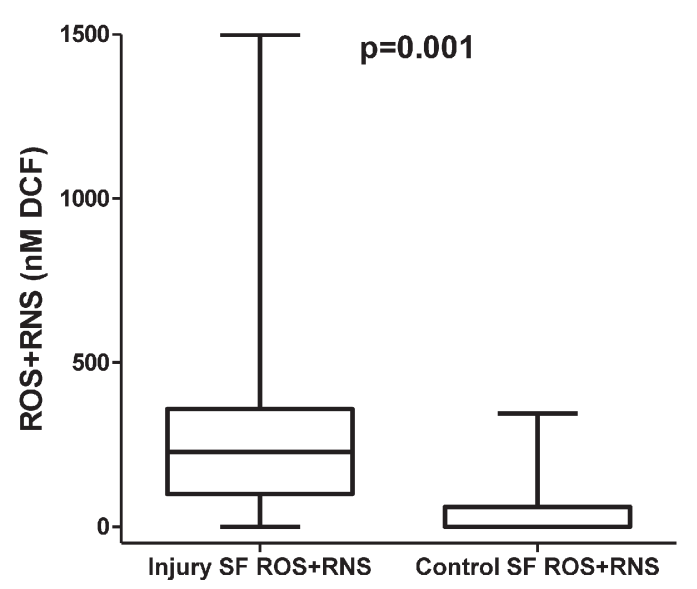

B

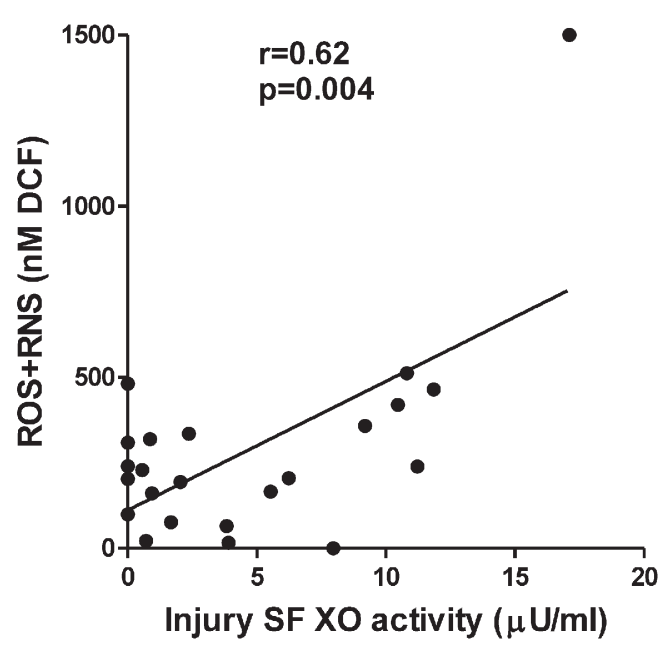

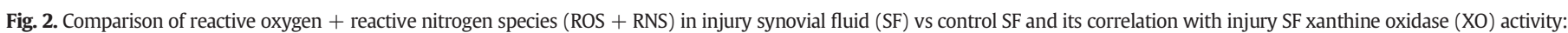

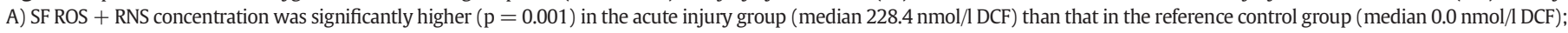

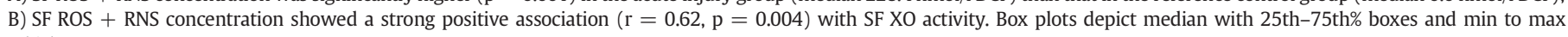
whiskers. 
A

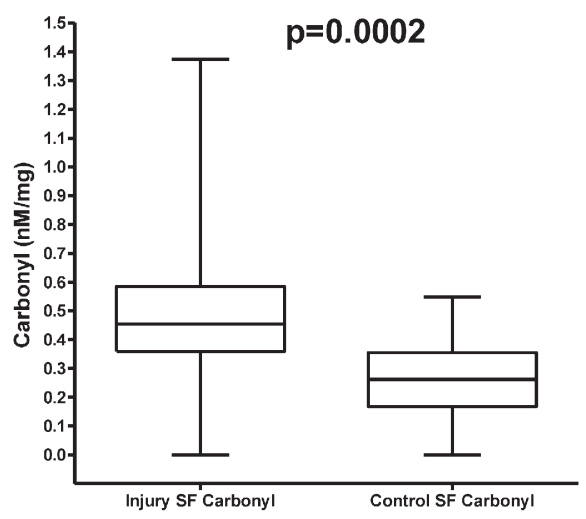

B

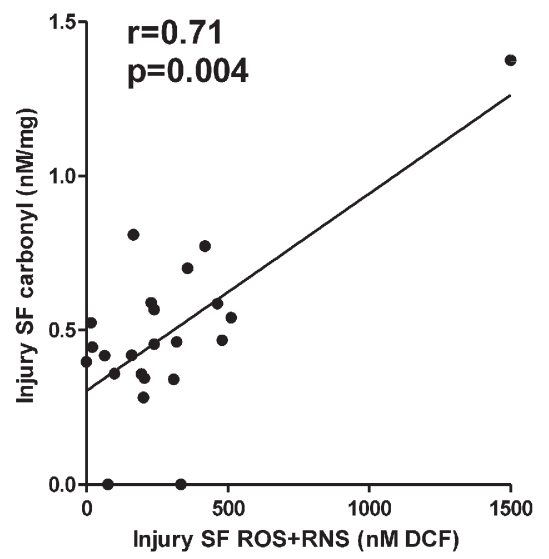

C

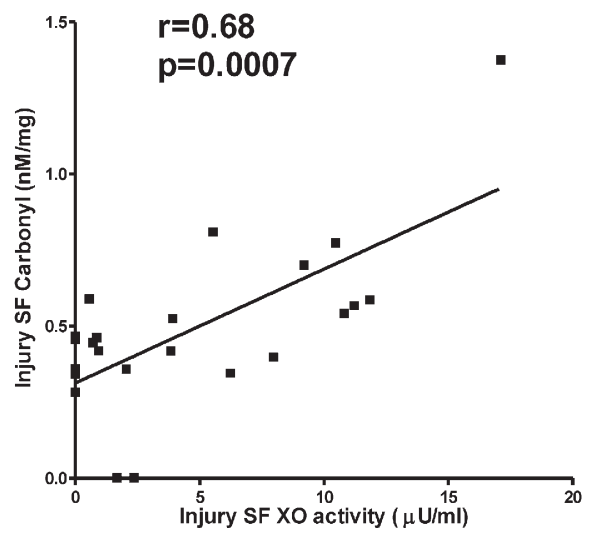

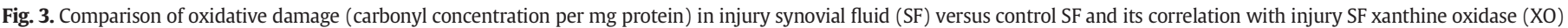

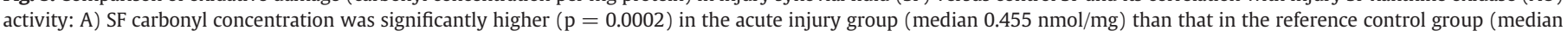

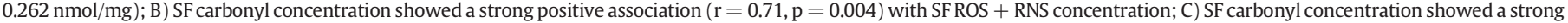
positive association $(r=0.68, p=0.0007)$ with SF XO activity. Box plots depict median with 25th-75th\% boxes and min to max whiskers.

be significantly elevated in the SF about 47 days after injury [36]. It is also possible that the rapid clearance of the very small CTx-II molecule from the joint might mask any increase. While CTxII is detectable in SF, it may be cleared more rapidly than CPII. While it has been reported that ROS also increase degradation of the cartilage matrix, either by direct effect or activation of collagenases [8], we did not observe a correlation of XO activity, ROS + RNS, or carbonyl with the degradation marker CTx-II. Again this lack of association is most likely due to the early timepoint, the rapid clearance of CTxII, or a combination of the two. Of note, even though CTx-II was not increased, we did find a relationship between it and CPII as expected for a coupled degradation and repair response to injury.

The dramatic increase seen in the pro-inflammatory cytokine IL-6 over the control group is consistent with previous reports of subjects with acute knee injury and significantly higher than has been reported for OA SF $[37,38]$. The lack of association of IL- 6 with any of the other analytes is not unexpected since IL-6 is part of a generalized acute phase response to injury.

The difference in age between the acute injury group (mean 39.4 years) and the reference control group (mean 64 years) was a limitation of this study; however, a young control group would be expected to have lower SF XO activity than our reference control group. Taken together this suggests that our results err on the side of conservatively estimating the increase in $\mathrm{XO}$ activity due to acute injury. Additionally in our analyses, all p values were adjusted for age. Another limitation of this study was the use of SF from OA subjects as a control group due to the difficulty of obtaining SF from non-arthritic subjects. The use of $\mathrm{SF}$ from knees contralateral to diseased knees but which themselves exhibit minimal or no OA should closely approximate normal joints. Furthermore, the very low levels of IL- 6 in this group were within the confidence interval of previous reports from normal SF controls and much lower than is seen in advanced OA, lending further credence to their use a control [37,38].

This study shows that SF XO activity is increased in acute joint injury and that this increase is strongly associated with an increase in the production of ROS + RNS as well as an increase in oxidative damage as measured by protein carbonyl content. These data confirm that oxidative stress participates in the pathophysiology of joint damage following acute joint injury. This increased XO activity was also associated with decreased type II collagen production suggesting that it may hinder the repair response of the acutely injured joint. While this is a small study, these results raise the intriguing possibility that an XOR inhibitor,
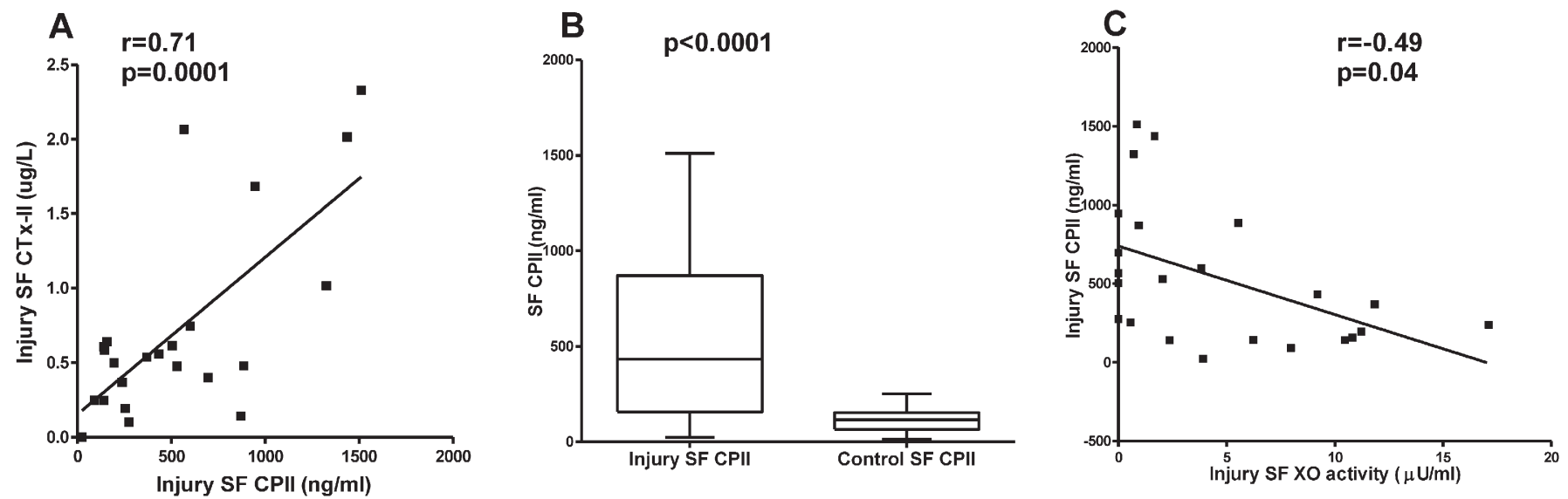

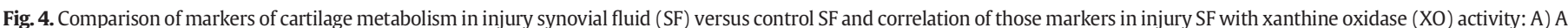

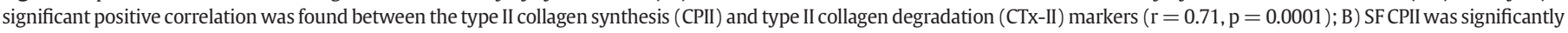

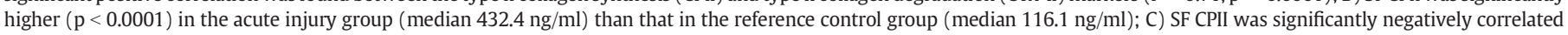
$(\mathrm{r}=-0.649, \mathrm{p}=0.04)$ with SF XO activity. Box plots depict median with 25th-75th\% boxes and min to max whiskers. 


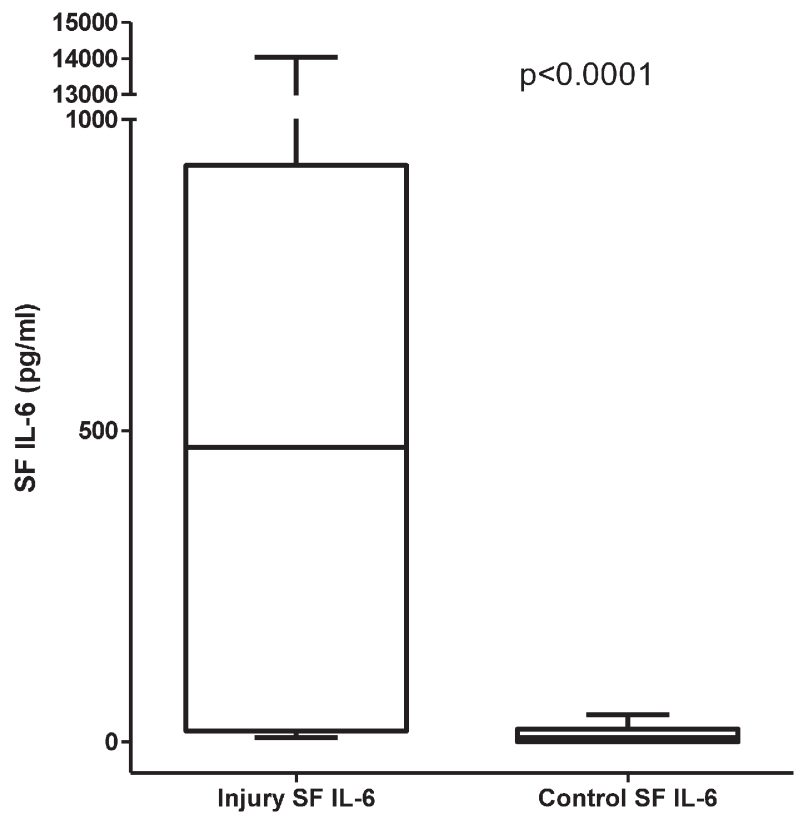

Fig. 5. Proinflammatory cytokine IL-6 in synovial fluid (SF): SF IL-6 was significantly higher $(\mathrm{p}<0.0001$ ) in the acute injury group (median $473.0 \mathrm{pg} / \mathrm{ml}$ ) than that in the reference control group (median $6.2 \mathrm{pg} / \mathrm{ml}$ ). Box plots depict median with 25 th-75th\% boxes and min to max whiskers.

such as allopurinol, may provide some therapeutic benefit for acute joint injury and help to mitigate the potential development of $\mathrm{OA}$ which typically occurs many years later [39].

\section{Acknowledgments}

This study was supported by the National Institute on Aging at the National Institutes of Health (\%P30 AG028716) (Claude D. Pepper Older Americans Independence Centers); and grants from the National Institute of Arthritis and Musculoskeletal and Skin Diseases at the National Institutes of Health (RO1 AR48769 and PO1 AR050245). Study sponsors had no involvement in study design, collection, analysis, and interpretation of the data, nor in manuscript preparation or submission.

\section{References}

[1] R. Hille, T. Nishino, Flavoprotein structure and mechanism. 4. Xanthine oxidase and xanthine dehydrogenase, FASEB J. 9 (1995) 995-1003.

[2] F. Stirpe, C.E. Della, The regulation of rat liver xanthine oxidase. Conversion in vitro of the enzyme activity from dehydrogenase (type D) to oxidase (type O), J. Biol. Chem. 244 (1969) 3855-3863.

[3] Y. Amaya, K. Yamazaki, M. Sato, K. Noda, T. Nishino, Proteolytic conversion of xanthine dehydrogenase from the NAD-dependent type to the 02-dependent type. Amino acid sequence of rat liver xanthine dehydrogenase and identification of the cleavage sites of the enzyme protein during irreversible conversion by trypsin, J. Biol. Chem. 265 (1990) 14170-14175.

[4] A. Kooij, H.J. Schiller, M. Schijns, C.J. Van Noorden, W.M. Frederiks, Conversion of xanthine dehydrogenase into xanthine oxidase in rat liver and plasma at the onset of reperfusion after ischemia, Hepatology 19 (1994) 1488-1495.

[5] R. Harrison, Structure and function of xanthine oxidoreductase: where are we now? Free Radic. Biol. Med. 33 (2002) 774-797.

[6] A. Meneshian, G.B. Bulkley, The physiology of endothelial xanthine oxidase: from urate catabolism to reperfusion injury to inflammatory signal transduction, Microcirculation 9 (2002) 161-175.

[7] C. Vorbach, R. Harrison, M.R. Capecchi, Xanthine oxidoreductase is central to the evolution and function of the innate immune system, Trends Immunol. 24 (2003) 512-517.

[8] Y.E. Henrotin, P. Bruckner, J.P. Pujol, The role of reactive oxygen species in homeostasis and degradation of cartilage, Osteoarthr. Cartil. 11 (2003) 747-755.

[9] W. Ehrlich, H. Huser, H. Kroger, Induction and activation of procollagenase in rabbit synovial fibroblasts after treatment with active oxygen released by xanthine/xanthine oxidase, Rheumatol. Int. 15 (1995) 131-136.
[10] D.A. Parks, D.N. Granger, Xanthine oxidase: biochemistry, distribution and physiology, Acta Physiol. Scand. Suppl. 548 (1986) 87-99.

[11] R.E. Allen, J.M. Outhwaite, C.J. Morris, D.R. Blake, Xanthine oxidoreductase is present in human synovium, Ann. Rheum. Dis. 46 (1987) 843-845.

[12] F. Hietbrink, L. Koenderman, G. Rijkers, L. Leenen, Trauma: the role of the innate immune system, World J. Emerg. Surg. 1 (2006) 15.

[13] M. Harbo, L. Bendix, A.C. Bay-Jensen, J. Graakjaer, K. Soe, T.L. Andersen, et al., The distribution pattern of critically short telomeres in human osteoarthritic knees, Arthritis Res. Ther. 14 (2012) R12.

[14] L.S. Lohmander, P.M. Englund, L.L. Dahl, E.M. Roos, The long-term consequence of anterior cruciate ligament and meniscus injuries: osteoarthritis, Am. J. Sports Med. 35 (2007) 1756-1769.

[15] V.B. Kraus, J. Birmingham, T.V. Stabler, S. Feng, D.C. Taylor, C.T. Moorman 3rd, et al. Effects of intraarticular IL1-Ra for acute anterior cruciate ligament knee injury: a randomized controlled pilot trial (NCT00332254), Osteoarthr. Cartil. 20 (2012) 271-278.

[16] S. Addison, R.E. Coleman, S. Feng, G. McDaniel, V.B. Kraus, Whole-body bone scintigraphy provides a measure of the total-body burden of osteoarthritis for the purpose of systemic biomarker validation, Arthritis Rheum. 60 (2009) 3366-3373.

[17] J.H. Kellgren, J.S. Lawrence, Radiological assessment of osteo-arthrosis, Ann. Rheum. Dis. 16 (1957) 494-502.

[18] M.H. Shamma'a, S.M. Nasrallah, U.A. al-Khalidi, Serum xanthine oxidase. An experience with 2000 patients, Am. J. Dig. Dis. 18 (1973) 15-22.

[19] M.G. Battelli, S. Musiani, M. Valgimigli, L. Gramantieri, F. Tomassoni, L. Bolondi, et al, Serum xanthine oxidase in human liver disease, Am. J. Gastroenterol. 96 (2001) 1194-1199.

[20] C. Ramboer, F. Piessens, J. De Groote, Serum xanthine oxidase and liver disease, Digestion 7 (1972) 183-195.

[21] R. Miesel, M. Zuber, Elevated levels of xanthine oxidase in serum of patients with inflammatory and autoimmune rheumatic diseases, Inflammation 17 (1993) 551-561.

[22] T. Yamamoto, Y. Moriwaki, S. Takahashi, Z. Tsutsumi, J. Yamakita, Y. Nasako, et al., Determination of human plasma xanthine oxidase activity by high-performance liquid chromatography, J. Chromatogr. B Biomed. Appl. 681 (1996) 395-400.

[23] J.E. Rinaldo, M. Clark, J. Parinello, V.L. Shepherd, Nitric oxide inactivates xanthine dehydrogenase and xanthine oxidase in interferon-gamma-stimulated macrophages, Am. J. Respir. Cell Mol. Biol. 11 (1994) 625-630.

[24] E. Tubaro, B. Lotti, C. Santiangeli, G. Cavallo, Xanthine oxidase increase in polymorphonuclear leucocytes and macrophages in mice in three pathological situations, Biochem. Pharmacol. 29 (1980) 1945-1948.

[25] R. Radi, H. Rubbo, K. Bush, B.A. Freeman, Xanthine oxidase binding to glycosaminoglycans: kinetics and superoxide dismutase interactions of immobilized xanthine oxidase-heparin complexes, Arch. Biochem. Biophys. 339 (1997) 125-135.

[26] E.E. Kelley, A. Trostchansky, H. Rubbo, B.A. Freeman, R. Radi, M.M. Tarpey, Binding of xanthine oxidase to glycosaminoglycans limits inhibition by oxypurinol, J. Biol. Chem. 279 (2004) 37231-37234.

[27] E.R. Stadtman, R.L. Levine, Free radical-mediated oxidation of free amino acids and amino acid residues in proteins, Amino Acids 25 (2003) 207-218.

[28] R.L. Levine, Carbonyl modified proteins in cellular regulation, aging, and disease Free Radic. Biol. Med. 32 (2002) 790-796.

[29] D. Li, G. Xie, W. Wang, Reactive oxygen species: the 2-edged sword of osteoarthritis Am. J. Med. Sci. (2012).

[30] Y. Henrotin, B. Kurz, T. Aigner, Oxygen and reactive oxygen species in cartilage degradation: friends or foes? Osteoarthr. Cartil. 13 (2005) 643-654.

[31] F. Nelson, L. Dahlberg, S. Laverty, A. Reiner, I. Pidoux, M. Ionescu, et al., Evidence for altered synthesis of type II collagen in patients with osteoarthritis, J. Clin. Invest. 102 (1998) 2115-2125

[32] L.S. Lohmander, M. Ionescu, H. Jugessur, A.R. Poole, Changes in joint cartilage aggrecan after knee injury and in osteoarthritis, Arthritis Rheum. 42 (1999) 534-544.

[33] V. Grishko, M. Xu, R. Ho, A. Mates, S. Watson, J.T. Kim, et al., Effects of hyaluronic acid on mitochondrial function and mitochondria-driven apoptosis following oxidative stress in human chondrocytes, J. Biol. Chem. 284 (2009) 9132-9139.

[34] R.H. Nielsen, R. Stoop, D.J. Leeming, M. Stolina, P. Qvist, C. Christiansen, et al., Evaluation of cartilage damage by measuring collagen degradation products in joint extracts in a traumatic model of osteoarthritis, Biomarkers 13 (2008) 79-87.

[35] T.L. Chmielewski, T.N. Trumble, A.M. Joseph, J. Shuster, P.A. Indelicato, M.W. Moser, et al., Urinary CTX-II concentrations are elevated and associated with knee pain and function in subjects with ACL reconstruction, Osteoarthr. Cartil. 20 (2012) 1294-1301.

[36] J.B. Catterall, T.V. Stabler, C.R. Flannery, V.B. Kraus, Changes in serum and synovial fluid biomarkers after acute injury (NCT00332254), Arthritis Res. Ther. 12 (2010) R229.

[37] A. Struglics, S. Larsson, N. Kumahashi, R. Frobell, L.S. Lohmander, Changes in cytokines and aggrecan ARGS neoepitope in synovial fluid and serum and in Cterminal crosslinking telopeptide of type II collagen and N-terminal crosslinking telopeptide of type I collagen in urine over five years after anterior cruciate ligament rupture: an exploratory analysis in the knee anterior cruciate ligament, nonsurgical versus surgical treatment trial, Arthritis Rheumatol. 67 (2015) 1816-1825.

[38] M. Beekhuizen, L.M. Gierman, W.E. van Spil, G.J. Van Osch, T.W. Huizinga, D.B. Saris, et al., An explorative study comparing levels of soluble mediators in control and osteoarthritic synovial fluid, Osteoarthr. Cartil. 21 (2013) 918-922.

[39] D. Simon, R. Mascarenhas, B.M. Saltzman, M. Rollins, B.R. Bach Jr., P. MacDonald, The relationship between anterior cruciate ligament injury and osteoarthritis of the knee, Adv. Orthop. 2015 (2015) 928301. 